\title{
Oligodendrogliomas in pediatric and adult patients: an outcome-based study from the Surveillance, Epidemiology, and End Result database
}

\author{
Christine SM Lau ${ }^{1,2}$ \\ Krishnaraj Mahendraraj' \\ Ronald S Chamberlain ${ }^{1-4}$ \\ 'Department of Surgery, Saint \\ Barnabas Medical Center, Livingston, \\ NJ, USA; '2Saint George's University \\ School of Medicine, Grenada, West \\ Indies; ${ }^{3}$ Department of Surgery, \\ New Jersey Medical School, Rutgers \\ University, Newark, NJ, ${ }^{4}$ Department \\ of Surgery, Banner MD Anderson \\ Cancer Center, Gilbert, AZ, USA
}

Correspondence: Ronald S Chamberlain Department of Surgery, Banner MD Anderson Cancer Center, 2940 East Banner Gateway Drive, Gilbert, AZ 85234, USA

Tel +l 4802564737

Fax +I 4802564734

Email ronald.chamberlain@bannerhealth. com

\author{
This article was published in the following Dove Press journal: \\ Cancer Management and Research \\ 2 May 2017 \\ Number of times this article has been viewed
}

Introduction: Oligodendrogliomas (OGs) account for $<20 \%$ of all intracranial tumors and $25 \%$ of gliomas. Despite improvements in imaging techniques allowing for earlier diagnosis, OG is rare among the pediatric population. This study examines a large cohort of OG patients in an effort to define the demographic, clinical, and pathologic factors associated with clinical and survival outcomes.

Methods: Data on 7,001 OG patients were abstracted from the Surveillance, Epidemiology, and End Result (SEER) database (1973-2013). Pediatric patients were defined as $\leq 19$ years old, and adult patients were defined as age $\geq 20$ years.

Results: Among 7,001 OG patients, $6.5 \%$ were pediatric (mean age $12 \pm 6$ years), and $93.5 \%$ were adult (mean age $46 \pm 15$ years). Overall, OGs were more common among males, with a male-to-female ratio of 1.28:1. Overall, OGs were more common among Caucasians $(76.9 \%)$ and also among the African American (10.8\% pediatric vs. $4.0 \%$ adult) and Hispanic $(12.8 \%$ pediatric vs. $11.8 \%$ adult). OGs occurred most commonly in the temporal lobe of pediatric patients and the frontal lobes of adults. Surgical resection was the primary treatment modality for both pediatric and adult populations ( $70.6 \%$ and $40.5 \%)$, followed by combined surgery and radiation (19.7\% and $41.2 \%$ ). Surgical resection was associated with significantly improved survival in both groups. Pediatric patients had a lower overall mortality (19.8\% vs. $48.5 \%)$ and lower cancer-specific mortality (17.6\% vs. $36.8 \%)$.

Conclusion: OGs most often present in Caucasian males in their fifth decade of life with tumors $>4 \mathrm{~cm}$ in size. Children typically present with tumors in the temporal lobe, while adults present with tumors in the frontal lobe. Surgical resection confers a survival advantage among all patients, especially pediatric patients. Overall survival (OS) and cancer-specific survival are higher in the pediatric population. Further studies on novel treatment techniques, including bevacizumab and immunotherapy approaches, are required.

Keywords: oligodendroglioma, brain cancer, SEER database

\section{Introduction}

The age-adjusted incidence of malignant brain and central nervous system (CNS) cancer is estimated at 3-4 per 100,000 population worldwide. ${ }^{1}$ In developed countries, the incidence is even higher, and in the US, it is $\sim 5$ cases per $100,000 .{ }^{1}$ With $>20,000$ new adult cases of malignant primary brain tumors diagnosed annually, $70 \%$ are malignant gliomas. ${ }^{1}$ Oligodendrogliomas (OGs) account for $<20 \%$ of all intracranial tumors and $25 \%$ of gliomas. ${ }^{1}$ 
Diagnosis of OGs remains difficult, and symptoms are related more to increased intracranial pressure, including headaches and other neurologic symptoms. ${ }^{2,3}$ More than half of all OG patients initially present with seizures, in which work-up ultimately leads to the diagnosis. In a retrospective study by Lee and Van ${ }^{3}$ involving 35 patients, $69 \%$ of OGs involved the frontal lobe, and seizure was the most common presenting symptom (74\%), followed by headaches (23\%). Creach et $\mathrm{al}^{4}$ reported similar results in 37 pediatric OG patients, and $62 \%$ of patients presented initially with seizures and $22 \%$ with headaches.

Radiological imaging demonstrates characteristic features of OGs, which aid in diagnosis. Computed tomography (CT) reveals hypoattenuating (57\%) or isoattenuating (23\%) lesions, often with calcifications and occasionally cystic degeneration and hemorrhage. ${ }^{2,3}$ Magnetic resonance imaging (MRI), which is superior to CT, allows for more accurate delineation of tumor involvement and demonstrates hypointense lesions on T1-weighted images and hyperintense lesions on T2-weighted images. ${ }^{2,3,5}$

On histopathological review, OGs are well-defined, soft, fleshy masses, which are pinkish gray in color. ${ }^{2,6}$ Gritty calcifications are common, and mucoid degeneration and leptomeningeal infiltration can also be seen., ${ }^{2,6}$ On microscopic examination, OGs are moderately cellular neoplasms with uniformly round, hyperchromatic nuclei surrounded by prominent clear cytoplasm known as a "perinuclear halo" and dense branching networks of capillaries resembling a "chicken-wire" pattern.,

Although there are no specific immunohistochemical markers pathognomonic of OGs, there are unique chromosomal abnormalities found often in these tumors. OGs typically display a loss of heterozygosity on the short arm of chromosome $1(1 \mathrm{p})$ and on the long arm of chromosome 19 (19q). ${ }^{2,4,5,7} \mathrm{Yu}$ et al ${ }^{7}$ studied 165 OG patients with known $1 \mathrm{p} / 19 \mathrm{q}$ status, of whom 95 patients had a $1 \mathrm{p} / 19 \mathrm{q}$ co-deletion, 12 patients had a $1 \mathrm{p}$ deletion alone, and 10 patients had a $19 q$ deletion alone.

Despite improvements in imaging techniques allowing for earlier diagnosis, OG remains rare among the pediatric population. Current knowledge regarding OG is limited, with most reports being retrospective observational studies that compare OG with other malignant brain tumors. In a retrospective study of 1,304 patients in Denmark by Nielsen et al, ${ }^{5}$ the incidence of OG has remained relatively stable while survival outcomes have been improving. OG in the pediatric population is much less prevalent than in adults, and knowledge about pediatric OG is finite, with very few studies having examined different treatment approaches and outcomes. Creach et al ${ }^{4}$ reported on survival outcomes among pediatric patients, but included only 37 patients at one hospital center. This study sought to examine a large cohort of pediatric and adult patients with OG from the Surveillance, Epidemiology, and End Results (SEER) database, in an effort to identify demographic, clinical, and treatment strategies that impact clinical outcomes. This study aims to delineate the differences among pediatric and adult OGs, by comparing demographic and tumor characteristics, treatment modalities utilized, as well as survival outcomes. Furthermore, this study reviews the current literature on treatment and survival outcomes for OGs in both pediatric and adult populations. By understanding these differences, this study can potentially guide therapeutic decision-making as well as provide a direction for future clinical trials.

\section{Methods}

Data for this study were extracted from the SEER database (1973-2013), which is part of the National Cancer Institute. Data from 18 SEER registries (Alaska Native Tumor Registry, Arizona Indians, Cherokee Nation, Connecticut, Detroit, Georgia Center for Cancer Statistics, Greater Bay Area Cancer Registry, Greater California, Hawaii, Iowa, Kentucky, Los Angeles, Louisiana, New Jersey, New Mexico, Seattle-Puget Sound, and Utah) were used and exported into SEER Stat software version 8.3.2.

Using the SEER International Classification of Disease for Oncology (ICD-O-3) codes 9450 and 9451, 7,001 confirmed cases of OGs were identified. Data were then exported to IBM SPSS ${ }^{\circledR}$ V23. Demographic and clinical data, including age, gender, race, primary site, tumor size, and type of treatment received (surgery, radiation, both, or no treatment), were extracted. Patients were grouped into pediatric patients (age $\leq 19$ years) and adult patients (age $\geq 20$ years). Patients with nonspecific site of tumor origin and those without histologic confirmation of cancer were excluded from the final study cohort used for analysis. The clinical outcomes examined were 1-, 2-, and 5-year overall survival (OS), mortality, and cancer-specific mortality. Categorical variables were compared using the chi-square test, whereas continuous variables were compared using Student's $t$-test and analysis of variance (ANOVA). Multivariate analysis was performed, and odds ratios (ORs) were calculated to determine factors that are independently associated with mortality and survival. Calculations including frequencies and multivariate analysis were conducted based on all available reported data (Tables 1-3). Long-term actuarial survival between groups 
Table I Demographic profiles of 7,00I patients with oligodendrogliomas from the SEER database, 1973-2013

\begin{tabular}{|c|c|c|c|c|}
\hline Variables & Overall & Pediatric & Adult & $p$-value \\
\hline $\mathrm{N}(\%)$ & 7,001 & $455(6.5)$ & $6,546(93.5)$ & - \\
\hline $\begin{array}{l}\text { Mean age, years (mean } \\
\pm \text { SD) }\end{array}$ & $42 \pm 17$ & $12 \pm 6$ & $46 \pm 15$ & - \\
\hline \multicolumn{5}{|l|}{ Gender, N (\%) } \\
\hline Male & $3,927(56.1)$ & $248(54.5)$ & $3,679(56.2)$ & 0.484 \\
\hline Female & $3,074(43.9)$ & $207(45.5)$ & $2,867(43.8)$ & 0.478 \\
\hline \multicolumn{5}{|l|}{ Race, N (\%)** } \\
\hline Caucasian & $5,348(76.9)$ & $317(70.0)$ & $5,031(77.4)$ & $<0.001$ \\
\hline African American & $312(4.5)$ & $49(10.8)$ & $263(4.0)$ & $<0.001$ \\
\hline Asian/Pacific Islander & $413(5.9)$ & $19(4.2)$ & $394(6.1)$ & 0.105 \\
\hline Hispanic & $824(11.8)$ & $58(12.8)$ & $766(11.8)$ & 0.518 \\
\hline $\begin{array}{l}\text { American Indian/ } \\
\text { Alaskan Native }\end{array}$ & $60(0.9)$ & $10(2.2)$ & $50(0.7)$ & $<0.001$ \\
\hline
\end{tabular}

Note: **Data presented for patients with available information only. Abbreviations: SEER, Surveillance, Epidemiology, and End Results; N, number; SD, standard deviation.

Table 2 Tumor characteristics and treatment for 7,00I patients with oligodendrogliomas from the SEER database, 1973-2013

\begin{tabular}{|c|c|c|c|c|}
\hline Variables & Overall & Pediatric & Adult & $p$-value \\
\hline $\mathrm{N}(\%)$ & 7,001 & $455(6.5)$ & $6,546(93.5)$ & - \\
\hline \multicolumn{5}{|l|}{ Primary site, $\mathrm{N}(\%)^{* *}$} \\
\hline Occipital lobe & $124(1.9)$ & $13(3.5)$ & III (I.8) & 0.01 \\
\hline Parietal lobe & $74 \mid(1 I .2)$ & $59(15.6)$ & $682(10.9)$ & $<0.001$ \\
\hline Temporal lobe & I,229 (I8.5) & $136(36.1)$ & I,093 (I7.5) & $<0.001$ \\
\hline Frontal lobe & $3,491(52.6)$ & II 4 (30.2) & $3,377(53.9)$ & $<0.001$ \\
\hline Overlapping lesion & $790(11.9)$ & $33(8.8)$ & $757(12.1)$ & 0.08 \\
\hline Brain, NOS & $260(3.9)$ & $22(5.8)$ & $238(3.8)$ & 0.03 \\
\hline \multicolumn{5}{|l|}{ Tumor size, $\mathrm{N}(\%)^{* *}$} \\
\hline Under $2 \mathrm{~cm}$ & $198(8.3)$ & $29(15.8)$ & $169(7.6)$ & $<0.001$ \\
\hline $2-4 \mathrm{~cm}$ & $963(40.3)$ & $104(56.8)$ & $859(39.0)$ & $<0.001$ \\
\hline Over $4 \mathrm{~cm}$ & I,227 (5। .4) & $50(27.3)$ & I, I 77 (53.4) & $<0.001$ \\
\hline \multicolumn{5}{|l|}{ Tumor grade, $\mathrm{N}(\%)$} \\
\hline Stage II & $5,245(74.9)$ & $410(90.1)$ & $4,835(73.9)$ & $<0.001$ \\
\hline Stage III & I,756 (25.I) & $45(9.9)$ & I,7II (26.I) & $<0.001$ \\
\hline \multicolumn{5}{|l|}{ Treatment, $\mathrm{N}(\%)^{* *}$} \\
\hline Neither & $675(9.8)$ & $30(6.6)$ & $645(10.0)$ & 0.02 \\
\hline Radiation only & $544(7.9)$ & $14(3.1)$ & $530(8.3)$ & $<0.001$ \\
\hline Surgery only & $2,917(42.5)$ & $319(70.6)$ & $2,598(40.5)$ & $<0.001$ \\
\hline Both & $2,733(39.8)$ & $89(19.7)$ & $2,644(4 I .2)$ & $<0.001$ \\
\hline
\end{tabular}

Note: **Data presented for patients with available information only.

Abbreviations: SEER, Surveillance, Epidemiology, and End Results; N, number; NOS, not otherwise specified.

was conducted using Kaplan-Meier analysis. Results were considered statistically significant at $p<0.05$.

\section{Ethical approval}

Ethical approval was obtained from Saint Barnabas Medical Center. This is a retrospective database study using the SEER database, no written informed consent was required as no individual patient identifiable information was utilized.

\section{Results}

\section{Demographic data}

A total of 7,001 cases of OG were reported in the SEER database between 1973 and 2013 (Figure 1). The mean age at diagnosis was $42 \pm 17$ years. There were significantly more adult patients than pediatric patients ( $93.5 \%$ vs. $6.5 \%$ ). In all, $93.5 \%$ of cases $(\mathrm{N}=6,546)$ were adults with a mean age at diagnosis of $46 \pm 15$ years, and $6.5 \%$ of cases $(\mathrm{N}=455)$ were pediatric patients with a mean age at diagnosis of $12 \pm 6$ years (Table 1 ).

The majority of cases of $\mathrm{OG}(\mathrm{N}=5,348 ; 76.9 \%)$ occurred in Caucasians, followed by Hispanics ( $\mathrm{N}=824 ; 11.8 \%)$, Asian, Pacific Islander ( $\mathrm{N}=413 ; 5.9 \%)$, African Americans $(\mathrm{N}=312$; $4.5 \%$ ), and American Indian/Alaskan Natives ( $\mathrm{N}=60 ; 0.9 \%)$. OG was more common among Caucasians in all age groups ( $70.0 \%$ of pediatric and $77.4 \%$ of adults, $p=0.001$ ); however, there were significantly more African Americans among the pediatric group ( $10.8 \%$ vs. $4.0 \%$ adult, $p<0.001)$.

Among all 7,001 OG patients, $56.1 \%$ were male $(\mathrm{N}=3,927)$ and $43.9 \%$ were female ( $\mathrm{N}=3,074)$, resulting in a male-tofemale ratio of 1.28:1. OG was more common among males in both the pediatric and adult population $(54.5 \%$ and $56.2 \%$, $p=0.48$ ). Among 455 pediatric patients, $54.5 \%$ were males $(\mathrm{N}=248)$ and $45.5 \%$ were females $(\mathrm{N}=207)$, resulting in a maleto-female ratio of 1.20:1. Among the 6,546 adult patients with OG, $56.2 \%$ were males $(\mathrm{N}=3,679)$ and $43.8 \%$ were females $(\mathrm{N}=2,867)$, resulting in a male-to-female ratio of $1.28: 1$.

\section{Tumor characteristics}

Primary tumor location was reported for 6,635 cases. Overall, more than half of all OG (52.6\%) occurred in the frontal lobe, $p<0.001$ (Table 2). The most common location for pediatric OG was the temporal lobe (36.1\%), whereas the most common location for adult OG was the frontal lobe (53.9\%).

Tumor size was available for 2,388 cases. In all, $51.4 \%$ $(\mathrm{N}=1,227)$ of all OGs were $>4 \mathrm{~cm}, 40.3 \%(\mathrm{~N}=963)$ were $2-4 \mathrm{~cm}$, and $8.3 \%(\mathrm{~N}=198)$ were $<2 \mathrm{~cm}$. A greater percentage of adult patients had tumor sizes greater than $4 \mathrm{~cm}(53.4 \% \mathrm{vs}$. $27.3 \%, p<0.001)$ compared with pediatric patients. Conversely, more pediatric patients had tumors between 2 and $4 \mathrm{~cm}(56.8 \%$ vs. $39.0 \%, p<0.001)$ compared with adult patients.

In all, $74.9 \%$ of the tumors were World Health Organization (WHO) Stage II tumors, while $25.1 \%$ were Stage III tumors. Significantly more pediatric tumors were Stage II ( $90.1 \%$ vs. $73.9 \%, p<0.001)$, while significantly more adult tumors were Stage III ( $26.1 \%$ vs. $9.9 \%, p<0.001)$. When stratified by tumor site, there was no significant difference among tumor size. 
Table 3 Survival outcomes of 7,00I patients with oligodendrogliomas from the SEER database, 1973-2013

\begin{tabular}{|c|c|c|c|c|}
\hline Variables & Overall & Pediatric & Adult & $p$-value \\
\hline $\bar{N}(\%)$ & 7,001 & $455(6.5)$ & $6,546(93.5)$ & - \\
\hline Mean overall survival, years (mean $\pm \mathrm{SD}$ ) & $15.319 \pm 0.324$ & $30.281 \pm 1.096$ & $13.597 \pm 0.305$ & $<0.001$ \\
\hline \multicolumn{5}{|l|}{ Actuarial survival by treatment, years** } \\
\hline Neither & $13.860 \pm 1.126$ & $28.294 \pm 3.021$ & $1 \mathrm{I} .345 \pm 0.727$ & $<0.001$ \\
\hline Radiation only & $8.600 \pm 0.492$ & $9.590 \pm 3.603$ & $8.490 \pm 0.496$ & $<0.001$ \\
\hline Surgery only & $20.185 \pm 0.607$ & $30.782 \pm 1.610$ & $18.179 \pm 0.642$ & $<0.001$ \\
\hline Both & $12.427 \pm 0.385$ & $22.295 \pm 2.121$ & $11.429 \pm 0.339$ & $<0.001$ \\
\hline \multicolumn{5}{|l|}{ Actuarial survival by primary site, years** } \\
\hline Occipital lobe & $15.692 \pm 1.582$ & & & \\
\hline Parietal lobe & $14.376 \pm 0.748$ & & & \\
\hline Temporal lobe & $15.59 \mid \pm 0.652$ & & & \\
\hline Frontal lobe & $14.538 \pm 0.399$ & & & \\
\hline \multicolumn{5}{|l|}{ Actuarial survival by size, years** } \\
\hline Under $2 \mathrm{~cm}$ & $23.790 \pm 0.709$ & & & \\
\hline $2-4 \mathrm{~cm}$ & $24.156 \pm 0.603$ & & & \\
\hline Over $4 \mathrm{~cm}$ & $21.412 \pm 0.575$ & & & \\
\hline \multicolumn{5}{|l|}{ Overall mortality, N (\%) } \\
\hline Alive & $3,739(53.4)$ & $365(80.2)$ & $3,374(51.5)$ & $<0.001$ \\
\hline Dead & $3,262(46.6)$ & $90(19.8)$ & $3,172(48.5)$ & $<0.001$ \\
\hline \multicolumn{5}{|l|}{ Cancer-specific mortality, N (\%) } \\
\hline Alive & $3,739(53.4)$ & $365(80.2)$ & $3,374(5 I .5)$ & $<0.001$ \\
\hline Cancer death & $2,489(35.6)$ & $80(17.6)$ & $2,409(36.8)$ & $<0.001$ \\
\hline Non cancer death & $773(11.0)$ & $10(2.2)$ & $763(11.7)$ & $<0.001$ \\
\hline \multicolumn{5}{|l|}{ Cumulative survival (\%) } \\
\hline I year & & 94 & 88 & $<0.001$ \\
\hline 2 years & & 89 & 75 & $<0.001$ \\
\hline 5 years & & 85 & 66 & $<0.001$ \\
\hline
\end{tabular}

Note: **Data presented for patients with available information only.

Abbreviations: SEER, Surveillance, Epidemiology, and End Results; N, number; SD, standard deviation.

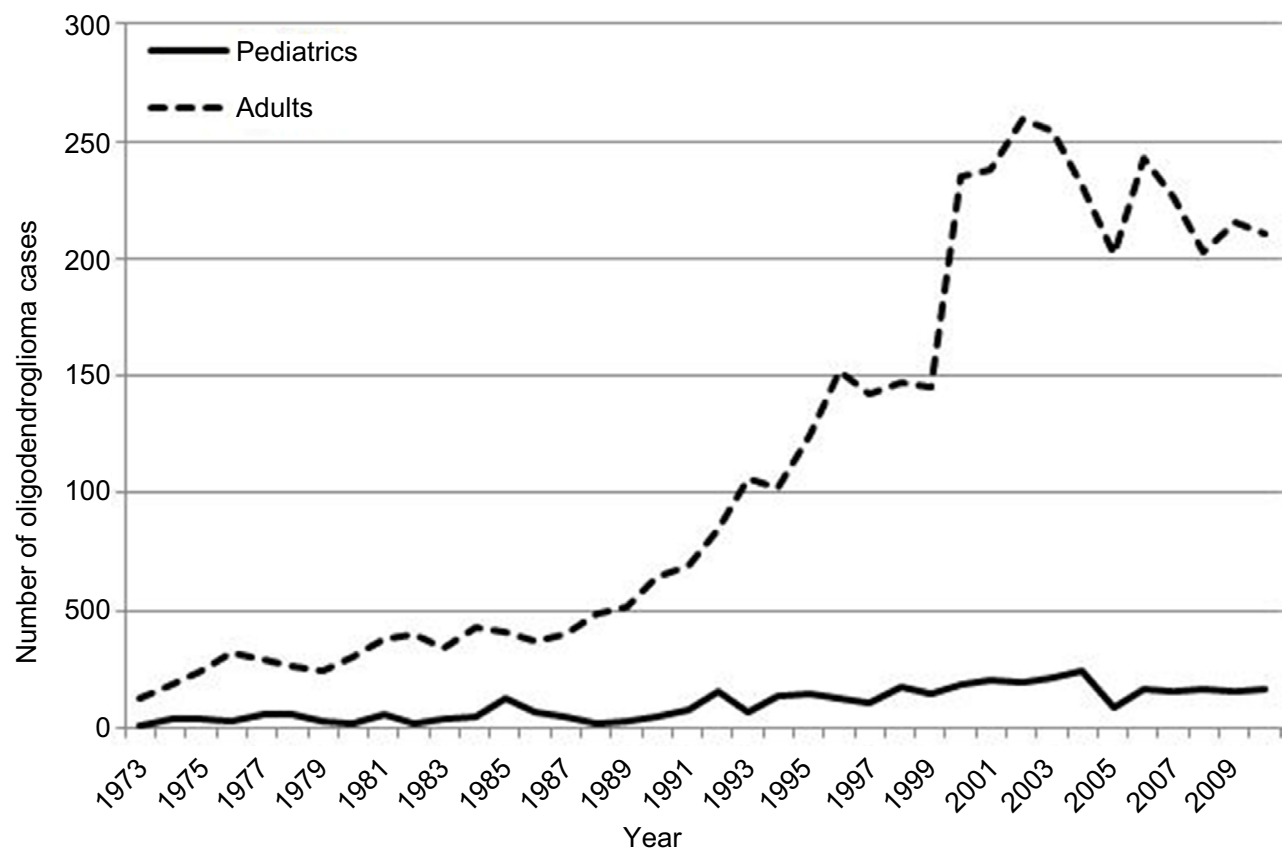

Figure I Oligodendroglioma incidence rates from the Surveillance, Epidemiology, and End Results (SEER) database, I973-2013. 


\section{Treatment and survival}

The type of treatment received was reported for 6,869 cases. In all, $42.5 \%(\mathrm{~N}=2,917)$ of all $\mathrm{OG}$ patients were treated with surgery alone, while $39.8 \%(\mathrm{~N}=2,733)$ of patients received combined surgery and radiation. In all, 7.9\% $(\mathrm{N}=544)$ of patients were treated with radiation alone, and $9.8 \%(\mathrm{~N}=675)$ received no treatment (Table 3). Most patients in all age groups received surgery as part of their treatment plan, either alone or in combination with radiation $(90.3 \%$ of pediatric and $81.7 \%$ of adults). More pediatric patients received surgery as primary treatment $(70.6 \%$ vs. $40.5 \%)$, while more adult patients received combined surgery and radiation $(41.2 \%$ vs. $19.7 \%)$ compared with pediatric patients, $p<0.001$. Patients who received surgery as the primary modality of treatment experienced significant survival benefit (mean survival $20.185 \pm 0.607$ years), as compared to those who received combined surgery and radiation treatment $(12.427$ \pm 0.385 years $)$, primary radiation treatment only $(8.600 \pm$ 0.492 years), or no treatment ( $13.860 \pm 1.126$ years, $p<0.001)$. Survival following surgery was significantly higher among the pediatric population in comparison to the adult population ( $30.782 \pm 1.610$ years vs. $18.179 \pm 0.642$ years, $p<0.001)$. Combined surgery and radiation was also associated with a significantly improved survival in pediatric patients (22.295 \pm 2.121 years vs. $11.429 \pm 0.339$ years, $p<0.001$ ), as compared to adult patients.

\section{Outcomes}

The mean OS for all OG patients was $15.3 \pm 0.3$ years. OS was significantly longer among the pediatric patients ( 30.3 \pm 1.1 years vs. $13.6 \pm 0.3$ years, $p<0.001)$. No significant difference in survival based on size or location of tumor was observed. For all OG patients, the overall and cancer-specific mortalities were found to be $46.6 \%$ and $35.6 \%$, respectively. Overall and cancer-specific mortalities among the pediatric patients (19.8\% and $17.6 \%$, respectively) were significantly lower than those in the adult patients $(48.5 \%$ and $36.8 \%$, $p<0.001$ ). Cumulative 1-, 2-, and 5-year survival rates were significantly higher among the pediatric patients, compared to those in the adult patients $(94 \%, 89 \%$, and $85 \%$ for pediatric patients vs. $88 \%, 75 \%$, and $66 \%$ for adult patients, $p<0.001$ ). Kaplan-Meier curves demonstrated significantly improved survival for the pediatric population compared with the adult population (Figure 2).

\section{Multivariate analysis}

Multivariate analysis identified WHO Grade III tumors (OR 1.61; 95\% CI, 1.44-1.80) and radiation therapy (OR

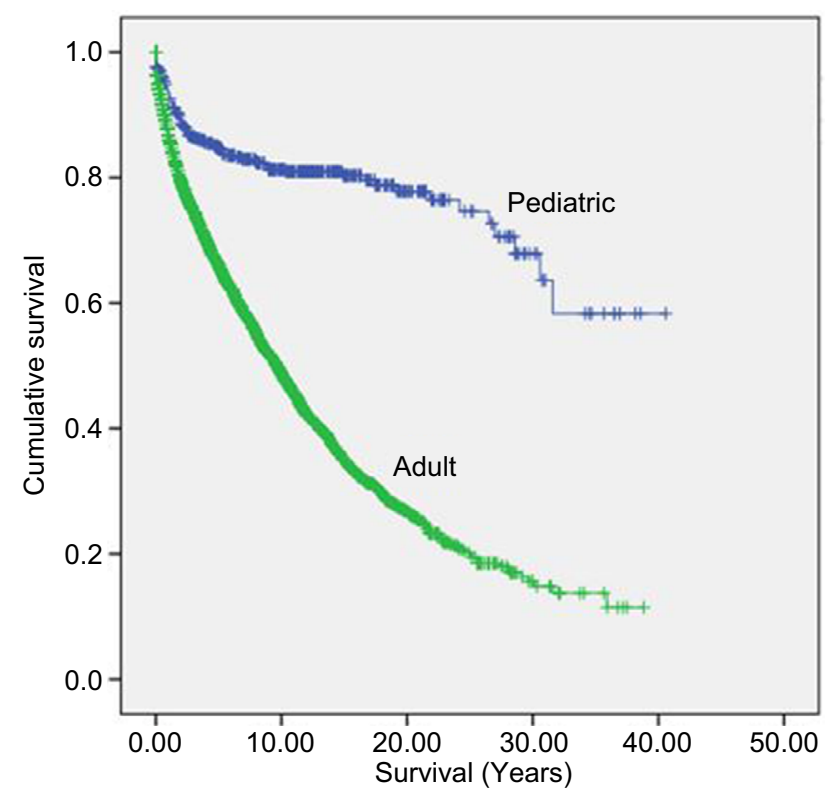

Figure 2 Kaplan-Meier actuarial survival curves for 7,002 patients with oligodendrogliomas from the Surveillance, Epidemiology, and End Results (SEER) database, 1973-2013.

2.33; 95\% CI, 1.83-2.96) as independently associated with increased mortality, while female gender (OR $0.88 ; 95 \% \mathrm{CI}$, $0.50-0.96)$ conferred survival advantage, $p<0.05$.

\section{Discussion}

OGs, first recognized by Bailey and Cushing in 1926, are the third most common glial neoplasm. ${ }^{2}$ Although they account for $2-5 \%$ of all primary brain tumors, they are extremely rare malignancies in the pediatric population, encompassing less than $1 \%$ of all pediatric CNS neoplasms. ${ }^{2}$ OGs occur most commonly in adult Caucasians in their fifth decade of life with slight male predominance. Pediatric OGs account for $<10 \%$ of all OG cases with a smaller male predominance. On multivariate analysis, female gender was associated with a survival advantage. Nielsen et $\mathrm{al}^{5}$ have suggested that female sex hormones may have a protective effect on the development of OGs. Fleury et al reported that OGs are frequently diagnosed later in life, with the age of diagnosis peaking later among females than men, further supporting this hypothesis. ${ }^{5,8}$ OGs are slow progressing tumors most commonly found in the frontal lobe of adults and the temporal lobe of children. Although most OGs were diagnosed as Stage II tumors, a significantly greater number of adult patients presented with Stage III anaplastic OGs compared to children.

Surgical resection remains first-line therapy for OGs and is associated with significantly improved survival rates in all age groups, especially among children and adolescents. The 
extent of surgical resection can greatly affect quality of life and survival outcomes, with longer survival times achieved following gross total resection. Shaw et $\mathrm{al}^{9}$ retrospectively analyzed 82 OG patients and reported prolonged median survival time following gross total resection compared to subtotal resection (12.6 years vs. 4.9 years). Among patients who received gross total resection, $21 \%$ were $<20$ years of age and had lower grade tumors $(37 \%)$, compared to those who received subtotal resection $(3 \%$ were $<20$ years of age and $14 \%$ had low-grade tumors). ${ }^{9}$ Multivariate analysis identified that frontal and parietal lobe tumor locations were associated with improved survival. ${ }^{9}$ Creach et al ${ }^{4}$ studied 37 pediatric OG patients, and gross total resection was associated with significantly better 5-year progression-free survival (PFS) (100\% vs. $28 \%, p=0.03)$ and median time to progression ( 9.75 years vs. 1.5 years), compared to subtotal resection. Snyder et al ${ }^{10}$ studied 93 patients with Grade II OGs and found that the patients with $>90 \%$ tumor resection experienced significantly longer 5-year OS (90\% vs. 87\%, $p=0.005)$ and PFS (62\% vs. $41 \%, p=0.004)$, compared to those who had $<90 \%$ tumor resection.

Given the difficulty in achieving gross total resection and decreased PFS experienced with subtotal resection, adjuvant radiation has been utilized to improve local control. In this study, radiation alone or as adjunct therapy was used more often in adult patients than pediatric patients; however, it conferred no survival benefit. Similarly, Rusthoven et al ${ }^{11}$ reported no survival advantage with adjuvant radiation therapy in OG patients, unlike patients with glioblastoma multiforme, anaplastic astrocytomas, and anaplastic oligoastrocytoma. The authors reported lower median survivals in OG patients receiving adjuvant radiation therapy, compared to patients receiving surgery alone (60 months vs. 78 months, $p=0.277) .{ }^{11}$ The 2 -year (67\% vs. $70 \%$ surgery alone, $p=0.277)$ and 5 -year (50\% vs. 56\% surgery alone, $p=0.277$ ) OSs were also decreased among patients receiving adjuvant radiation. ${ }^{11}$ The European Organization for Research and Treatment of Cancer (EORTC) 22845 trial by van den Bent et $\mathrm{al}^{12}$ randomized patients with astrocytomas and OGs to receive either early adjuvant radiation therapy or delayed adjuvant radiation therapy initiated at the time of tumor recurrence. Early adjuvant radiation therapy prolonged median PFS (5.3 years vs. 3.4 years, $p<0.0001$ ), but OS remained similar ( 7.4 years vs. 7.2 years, $p=0.872){ }^{12}$ Although radiation has proved beneficial in prolonging PFS, especially in low-grade OGs with residual tumor following resection and high-grade OGs, the optimal timing and dose of radiation remain controversial and poorly defined. ${ }^{11}$
Although chemotherapy data are not reported in the SEER database and were therefore not included in this study, adjuvant chemotherapy for the treatment of OG was initially evaluated in a report by Cairncross and Macdonald who noted favorable chemotherapeutic responses in eight OG patients receiving procarbazine, lomustine, and vincristine (PCV). ${ }^{2,13}$ A Phase II study by the National Cancer Institute of Canada Clinical Trials Group reported a 75\% response rate for PCV, with a median time to progression of 25.2 months among complete responders and 14.2 months among partial responders. ${ }^{14}$ In the NOA-04 randomized controlled trial (RCT) by Wick et al ${ }^{15}$ involving 274 patients randomly assigned to receive initial chemotherapy $(\mathrm{N}=135)$ or initial radiation therapy $(\mathrm{N}=139)$, there was no significant difference in PFS (31.9 months vs. 30.6 months, $p>0.05$ ) or OS (82.6 months vs. 72.1 months, $p>0.05$ ).

The use of chemotherapy combined with radiation therapy has also been extensively studied in OG. The Radiation Therapy Oncology Group (RTOG) Trial 9402 reported by Cairncross et $\mathrm{al}^{16}$ randomized patients with anaplastic OGs and anaplastic oligoastrocytomas to receive either PCV plus radiation therapy $(\mathrm{N}=142)$ or radiation therapy alone $(\mathrm{N}=142)$ and reported increased PFS with the use of adjuvant chemotherapy ( 2.6 years vs. 1.7 years, $p=0.04$ ) with most benefit seen among patients with $1 \mathrm{p} / 19 \mathrm{q}$ co-deletion ( 4.0 years vs. 1.4 years, $p<0.001)$. However, OS was similar in both groups (4.9 years vs. 4.7 years, $p=0.26$ ). The EORTC Study 26951 published by van den Bent et al ${ }^{17,18}$ randomized patients with anaplastic OGs and anaplastic oligoastrocytomas to receive either PCV plus radiation therapy $(\mathrm{N}=185)$ or radiation therapy alone $(\mathrm{N}=183)$ and reported significantly increased PFS (24.3 months vs. 13.2 months, $p<0.05)$ and OS (42.3 vs. 30.6 months, $p<0.05$ ) with the use of adjuvant PCV. Furthermore, the study showed that adjuvant $\mathrm{PCV}$ had a much greater improvement on PFS in patients with $1 p / 19 q$ codeletion (157 months vs. 50 months), compared to patients without the chromosomal deletions (15 months vs. 9 months). ${ }^{17,18}$ Adjuvant PCV also had greater improvements on PFS in patients with methylguanine-DNA methyltransferase (MGMT) methylation (70.9 months vs. 43.3 months), compared to patients without the MGMT methylation (16.3 months vs. 15.6 months).${ }^{18}$ Adjuvant PCV also had greater improvements on PFS in patients with isocitrate dehydrogenase (IDH) mutation (PFS not yet reached at 140 months vs. 64.8 months), compared to wild IDH (19.0 months vs. 14.7 months). ${ }^{18}$

More recently, temozolomide (TMZ) has demonstrated very promising results as a chemotherapeutic alternative to $\mathrm{PCV}^{2}$ Although TMZ has slightly lower reported response 
rates compared to $\mathrm{PCV}$, it has significantly improved toxicity profile with fewer than $10 \%$ of patients receiving TMZ developing a grade 3 or 4 hematologic toxicity compared to $50 \%$ of patients receiving $\mathrm{PCV}^{7,19}$ Speirs et $\mathrm{al}^{20}$ studied 59 patients with anaplastic OG or anaplastic astrocytoma and reported superior OS with the use of radiation therapy and adjuvant $\mathrm{TMZ}$ among patients with $1 \mathrm{p} / 19 \mathrm{q}$ codeletion $(91 \%$ vs. $68 \%$ at 5 years, $p=0.02$ ), compared to patients without the chromosomal deletions. PFS was also improved but not statistically significant ( $70 \%$ vs. $46 \%$ at 5 years, $p=0.10$ ).

Alternative treatments, including bevacizumab and immunotherapy approaches, are currently under investigation. Chamberlain and Johnston conducted a retrospective study involving 22 patients with recurrent $1 \mathrm{p} 19 \mathrm{q}$ codeletion OG treated with single-agent bevacizumab after failing alkylator-based therapy and reported a median survival of 8.5 months and 6-month and 12-month PFS rates of $68 \%$ and $23 \%$. ${ }^{21}$ The recent AVAglio and RTOG-0825 Phase III RCTs reported prolonged PFS with the addition of bevacizumab to radiotherapy and TMZ in glioblastoma patients, but no improvement in OS. ${ }^{22,23}$ The RTOG-0825 RCT involving 321 patients demonstrated a PFS of 10.7 months in patients treated with bevacizumab in addition to radiotherapy and TMZ, compared to 7.3 months among patients not receiving bevacizumab, $p<0.01$. No significant difference in median survival time was observed (15.7 months vs. 16.1 months, $p=0.21) .{ }^{24}$ Immunotherapy has also begun to be studied in current ongoing animal studies. ${ }^{25}$ SOX-2, a transcription factor required for self-renewal of neural stem cells, is highly expressed in OGs. ${ }^{25}$ Favaro et $\mathrm{al}^{25}$ vaccinated mice with SOX-2 peptides, which were previously shown to elicit T-cell activation against SOX-2 expressing glioblastoma cell in vitro and reported significantly improved survival times in mice, which nearly doubled when combined with TMZ.

Although several factors have been associated with $\mathrm{OG}$ survival, tumor grade has shown the strongest association with survival, with low-grade tumors having improved OS. Age $<20$ years, frontal or parietal tumor location, presence of calcification, and gross total resection have also been associated with improved OS. Conversely, factors including seizures, temporal lobe tumors, and side of tumor did not affect survival outcomes. ${ }^{9}$ Similarly, this study demonstrated that tumor location did not impact survival. Surgical resection remains the treatment for $\mathrm{OG}$, while adjuvant radiation has a role in poorly and undifferentiated tumors and is associated with improved survival. Recent studies have also shown that IDH $1 / 2$ mutations have a significant impact on survival and outcomes. Over half of the OG patients have an IDH-1 mutation.
It has been hypothesized that these mutations decrease the cell's ability to produce nicotinamide adenine dinucleotide phosphate (NADPH), reducing its ability to scavenge oxygen species and making the tumor cells more susceptible radiation and chemotherapy. In a review by Chen et al, ${ }^{26}$ IDH mutations were associated with significantly prolonged OS (hazard ratio [HR] 0.358; 95\% CI, 0.264-0.487; $p<0.001)$ and PFS (HR $0.322 ; 95 \%$ CI, $0.242-0.455 ; p<0.001)$.

There are several limitations to this study given the retrospective nature of this database study. First, the SEER database did not accurately code for various clinical factors such as molecular or genetic characteristics, chromosomal $1 \mathrm{p}$ and/or $19 \mathrm{q}$ deletions, presenting symptoms, and method of diagnostic confirmation, which could have had an impact on survival. Second, information on diagnostic imaging and long-term follow-up were lacking. Data on surgical and radiation therapy were available in the SEER database; however, information on the extent of surgical resection achieved and chemotherapy received was not available, which limited this study's ability to evaluate the impact of adjuvant or neoadjuvant therapy. Furthermore, as newer treatment techniques are constantly being discovered, the treatment paradigm for OGs is changing. As radiation and chemotherapy techniques improve, and newer novel techniques including targeted therapy emerge, the treatment pattern changes and survival outcomes improve. These temporal trends, however, were not captured in this study. There may also be elements of selection bias, since SEER registries are more likely to sample from urban than from rural areas. Despite these limitations, the SEER database has data obtained from $26 \%$ of the US population, and these findings can be generalized to the overall population.

\section{Summary}

OGs among the pediatric population are rare and account for $<10 \%$ of all OG cases. Similar to adult OGs, pediatric OGs occur most commonly among Caucasian males. Pediatric OGs are exceedingly more common in the temporal lobe, while adult OGs tend to occur in the frontal lobe. Surgery is the treatment of choice for OGs and is associated with significantly prolonged survival, especially among pediatric patients. OS and cancer-specific survival are also higher in the pediatric population compared with their adult counterparts. Given the difficulty in achieve gross total resection and the low PFS associated with surgical resection, as well as the neurocognitive deficits and toxicities associated with adjuvant radiation and chemotherapy, further studies investigating adjuvant therapy and the role of radiation and chemotherapy 
in the treatment of pediatric OGs are required to optimize the management and survival in these patients.

\section{Disclosure}

The authors report no conflicts of interest in this work.

\section{References}

1. Ahmed R, Oborski MJ, Hwang M, Lieberman FS, Mountz JM. Malignant gliomas: current perspectives in diagnosis, treatment, and early response assessment using advanced quantitative imaging methods. Cancer Manag Res. 2014;6:149-170.

2. Koeller KK, Rushing EJ. From the archives of the AFIP: oligodendroglioma and its variants: radiologic-pathologic correlation. Radiographics. 2005;25(6):1669-1688.

3. Lee YY, Van TP. Intracranial oligodendrogliomas: imaging findings in 35 untreated cases. AJR Am J Roentgenol. 1989;152(2):361-369.

4. Creach KM, Rubin JB, Leonard JR, et al. Oligodendrogliomas in children. J Neurooncol. 2012;106(2):377-382.

5. Nielsen MS, Christensen HC, Kosteljanetz M, Johansen C. Incidence of and survival from oligodendroglioma in Denmark, 1943-2002. Neuro Oncol. 2009;11(3):311-317.

6. Engelhard HH, Stelea A, Cochran EJ. Oligodendroglioma: pathology and molecular biology. Surg Neurol. 2002;58(2):111-117.

7. Yu T, Kang HC, Lim DH, et al. Pattern of care of anaplastic oligodendroglioma and oligoastrocytoma in a Korean population: the Korean radiation oncology group study 13-12. JNeurooncol. 2015;121(3):531-539.

8. Fleury A, Menegoz F, Grosclaude P, et al. Descriptive epidemiology of cerebral gliomas in France. Cancer. 1997;79(6):1195-1202.

9. Shaw EG, Scheithauer BW, O'Fallon JR, Tazelaar HD, Davis DH. Oligodendrogliomas: the Mayo Clinic experience. J Neurosurg. 1992;76(3): 428-434.

10. Snyder LA, Wolf AB, Oppenlander ME, et al. The impact of extent of resection on malignant transformation of pure oligodendrogliomas. J Neurosurg. 2014;120(2):309-314.

11. Rusthoven CG, Carlson JA, Waxweiler TV, et al. The impact of adjuvant radiation therapy for high-grade gliomas by histology in the United States population. Int J Radiat Oncol Biol Phys. 2014;90(4):894-902.

12. van den Bent MJ, Afra D, de WO, et al. Long-term efficacy of early versus delayed radiotherapy for low-grade astrocytoma and oligodendroglioma in adults: the EORTC 22845 randomised trial. Lancet. 2005;366(9490):985-990.

13. Cairncross JG, Macdonald DR. Successful chemotherapy for recurrent malignant oligodendroglioma. Ann Neurol. 1988;23(4):360-364.
14. Cairncross G, Macdonald D, Ludwin S, et al. Chemotherapy for anaplastic oligodendroglioma. National Cancer Institute of Canada Clinical Trials Group. J Clin Oncol. 1994;12(10):2013-2021.

15. Wick W, Hartmann C, Engel C, et al. NOA-04 randomized phase III trial of sequential radiochemotherapy of anaplastic glioma with procarbazine, lomustine, and vincristine or temozolomide. J Clin Oncol. 2009;27(35):5874-5880.

16. Cairncross G, Berkey B, Shaw E, et al. Phase III trial of chemotherapy plus radiotherapy compared with radiotherapy alone for pure and mixed anaplastic oligodendroglioma: Intergroup Radiation Therapy Oncology Group Trial 9402. J Clin Oncol. 2006;24(18):2707-2714.

17. van den Bent MJ, Carpentier AF, Brandes AA, et al. Adjuvant procarbazine, lomustine, and vincristine improves progression-free survival but not overall survival in newly diagnosed anaplastic oligodendrogliomas and oligoastrocytomas: a randomized European Organisation for Research and Treatment of Cancer phase III trial. J Clin Oncol. 2006;24(18):2715-2722.

18. van den Bent MJ, Brandes AA, Taphoorn MJ, et al. Adjuvant procarbazine, lomustine, and vincristine chemotherapy in newly diagnosed anaplastic oligodendroglioma: long-term follow-up of EORTC brain tumor group study 26951. J Clin Oncol. 2013;31(3):344-350.

19. Jastaniyah N, Murtha A, Pervez N, et al. Phase I study of hypofractionated intensity modulated radiation therapy with concurrent and adjuvant temozolomide in patients with glioblastoma multiforme. Radiat Oncol. 2013;8:38.

20. Speirs CK, Simpson JR, Robinson CG, et al. Impact of $1 \mathrm{p} / 19 \mathrm{q}$ codeletion and histology on outcomes of anaplastic gliomas treated with radiation therapy and temozolomide. Int J Radiat Oncol Biol Phys. 2015; 91(2):268-276.

21. Chamberlain MC, Johnston S. Bevacizumab for recurrent alkylator-refractory anaplastic oligodendroglioma. Cancer. 2009;115(8):1734-1743.

22. Sandmann T, Bourgon R, Garcia J, et al. Patients with proneural glioblastoma may derive overall survival benefit from the addition of bevacizumab to first-line radiotherapy and temozolomide: retrospective analysis of the AVAglio trial. J Clin Oncol. 2015;33(25):2735-2744.

23. Chinot OL, de La Motte RT, Moore N, et al. AVAglio: phase 3 trial of bevacizumab plus temozolomide and radiotherapy in newly diagnosed glioblastoma multiforme. Adv Ther. 2011;28(4):334-340.

24. Gilbert MR, Dignam JJ, Armstrong TS, et al. A randomized trial of bevacizumab for newly diagnosed glioblastoma. NEngl J Med. 2014;370(8): 699-708.

25. Favaro R, Appolloni I, Pellegatta S, et al. Sox 2 is required to maintain cancer stem cells in a mouse model of high-grade oligodendroglioma. Cancer Res. 2014;74(6):1833-1844.

26. Chen JR, Yao Y, Xu HZ, Qin ZY. Isocitrate dehydrogenase (IDH) $1 / 2$ mutations as prognostic markers in patients with glioblastomas. Medicine (Baltimore). 2016;95(9):e2583.
Cancer Management and Research

\section{Publish your work in this journal}

Cancer Management and Research is an international, peer-reviewed open access journal focusing on cancer research and the optimal use of preventative and integrated treatment interventions to achieve improved outcomes, enhanced survival and quality of life for the cancer patient. The manuscript management system is completely online and includes

\section{Dovepress}

a very quick and fair peer-review system, which is all easy to use. Visit http://www.dovepress.com/testimonials.php to read real quotes from published authors. 